\title{
Dealing with hunger: Metabolic stress responses in tumors
}

\author{
Michael A. Reid, Mei Kong** \\ Department of Cancer Biology, Beckman Research Institute of City of Hope Cancer Center, Duarte, CA 91010, USA \\ E-mail: mekong@coh.org \\ *Corresponding author \\ Published: 30 September, 2013 \\ Journal of Carcinogenesis 2013,12:17 \\ Received: 13 May, 2013 \\ This article is available from: http://www.carcinogenesis.com/content//2/1/17 \\ Accepted: 06 August, 2013 \\ (c) 2013 Reid
}

\begin{abstract}
Increased nutrient uptake and usage is a hallmark of many human malignancies. During the course of tumorigenesis, cancer cells often outstrip their local nutrient supply leading to periods of nutrient deprivation. Interestingly, cancer cells often develop strategies to adapt and survive these challenging conditions. Accordingly, understanding these processes is critical for developing therapies that target cancer metabolism. Exciting new progress has been made in elucidating the mechanisms used by cancer cells under nutrient restricted conditions. In this review, we highlight recent studies that have brought insight into how cancer cells deal with low nutrient environments.
\end{abstract}

Keywords: AMP-activated protein kinase, metabolic stress, protein phosphatase 2A, p53

\section{INTRATUMORAL NUTRITIONAL STRESS}

A considerable body of evidence demonstrates that tumor cells display fundamental changes in metabolism and enhance nutrient uptake to meet increased bioenergetic demands of proliferation. ${ }^{[1]}$ In the 1920's, Otto Warburg published the seminal observation that cancer cells take up glucose at a surprisingly high rate and convert it primarily to lactate rather than oxidizing it completely, despite available oxygen. Recent studies suggest that this "Warburg effect" seen in cancer cells directly results from oncogenic mutations selected for during tumorigenesis. ${ }^{[2]}$ For example, the oncogene phosphoinositide 3-kinase is one of the most commonly mutated kinases in human cancer and plays a direct role in stimulating the conversion of cells from aerobic metabolism to glycolysis. Renewed interest in the Warburg

\begin{tabular}{|l|l|}
\hline \multicolumn{2}{|c|}{ Access this article online } \\
\hline Quick Response Code: & Website: \\
& www.carcinogenesis.com \\
\cline { 2 - 3 } & \\
\hline
\end{tabular}

effect has led to increased awareness that cancer cells also depend on a continued supply of glutamine for survival and proliferation. ${ }^{[3]}$ Glutamine uptake by transformed cells in culture is 10-fold greater than that of any other amino acid and glutamine is a key substrate required for anabolic growth of cancer cells. ${ }^{[4]}$ Glutamine is particularly important for highly proliferative cells because it provides a source of nitrogen for transamination reactions that maintain intracellular pools of non-essential amino acids and nucleotides. Glutamine catabolism can also provide nicotinamide adenine dinucleotide phosphate (NADPH) to maintain tricarboxylic acid cycle intermediates. ${ }^{[2]}$ Moreover, recent studies have demonstrated that glutamine levels play a critical role to activate mammalian target of rapamycin (mTOR) signaling for protein translation in cancer cells. ${ }^{[5]}$ Glutamine is also important for suppressing oxidative stress as glutamine can donate both carbon and nitrogen to glutathione, a major intracellular antioxidant. ${ }^{[6]}$ Similar to glucose metabolism, it has been recently demonstrated that increased uptake of glutamine is also controlled by oncogenes, such as $\mathrm{c}-\mathrm{Myc}$ and $\mathrm{K}-\mathrm{Ras}$. Oncogenic levels of $\mathrm{c}-\mathrm{Myc}$ are linked to increased glutaminolysis through coordinated transcriptional regulation of glutamine transporters and glutaminase enzymes while K-Ras promotes transcriptional 
reprogramming of key metabolic enzymes Glud1 and Got1 to promote growth of pancreatic ductal adenocarcinoma cells. $^{[3,7-9]}$

A paradox of glucose/glutamine addicted cancer cells is that they depend upon both nutrients for survival and proliferation; however, enhanced dependence on glucose/ glutamine metabolism often exceeds its production or depletes its local supply, resulting in tumor cells encountering nutrient deprived conditions. Although tumor cells have increased glucose uptake, it has been shown that glucose levels in bulk tumors can fall lower than those in normal tissues of the same tissue origins. ${ }^{[10]}$ Moreover, it was reported over 50 years ago that glutamine falls to almost undetectable levels in tumors compared with normal tissue. ${ }^{[1]]}$ Intriguingly, it has been demonstrated that glutamine levels are even lower in core regions of tumors compared with periphery. ${ }^{[12]}$ These observations suggest that tumors encounter low nutrient conditions in vivo and have developed adaptive mechanisms to sense, survive and thrive in low nutrient conditions.

In this review, we highlight recent studies involving nutrient sensing and downstream effector mechanisms important for adaptation under conditions of nutrient stress. The recent progress in the field of cancer metabolism provides novel concepts for testing the synergistic potential of combination therapies that target both signal transduction and metabolic pathways.

\section{NUTRIENT SENSING BY SIGNALING PATHWAYS}

\section{AMP-activated protein kinase}

The AMPK is an evolutionarily conserved heterotrimeric protein complex consisting of a catalytic $\alpha$ subunit and regulatory $\beta$ and $\gamma$ subunits. This complex plays a critical role in regulating stress responses as it senses changes in the cellular ratio of AMP to adenosine triphosphate (ATP). Upon activation, AMPK phosphorylates numerous substrates in order to increase cellular ATP levels by several mechanisms such as increasing glucose uptake, inhibiting gluconeogenesis and increasing mitochondrial biogenesis.

A key mechanism by which AMPK increases cellular energy is through inhibition of mTOR by direct phosphorylation of TSC2 and raptor, both negative regulators of mTOR [Figure 1]. ${ }^{[13,14]}$ As mTOR is the master regulator of protein synthesis and other anabolic pathways, its inhibition is essential for conserving energy under conditions of nutrient restriction. Another way AMPK directly inhibits protein translation is by activation of eukaryotic elongation factor

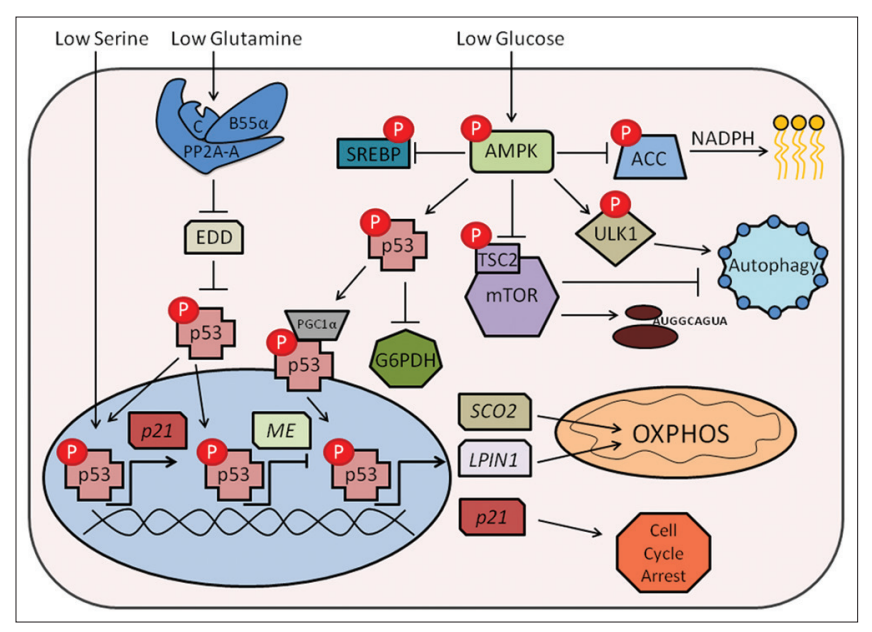

Figure I: Schematic representation of how cells respond to various metabolic stresses. Low levels of nutrients are detected by kinases and phosphatases, which modulate downstream effector proteins such as transcription factors to reprogram cellular functions and promote survival

2 kinase (eEF2), which phosphorylates and inactivates eukaryotic elongation factor. ${ }^{[15]}$ Interestingly, it was shown that inhibition of eEF2 via activation of AMPK is a conserved mechanism used by tumor cells in order to survive and adapt to periods of nutrient deprivation. ${ }^{[16]}$

Under conditions of nutrient stress, the inhibition of macromolecule biosynthesis may be insufficient to restore cellular energy levels. A major strategy for cells to scavenge energy precursors is through autophagy, a process by which cells recycle non-essential macromolecules and organelles to provide nutrients and energy. ${ }^{[17]}$ Although autophagy has many mechanism of regulation such as mTOR-directed inhibition, recent studies suggest that AMPK directly activates autophagy by phosphorylation of ULK1, an essential kinase for the initiation of autophagy [Figure 1]. ${ }^{[18]}$

Besides inhibition of protein translation and activation of autophagy, AMPK has been reported to promote cell survival through an adaptive cell cycle arrest mechanisms. Specifically, in response to low glucose levels, AMPK phosphorylates the transcription factor p53 [Figure 1]. AMPK-dependent activation of p53 allows cells to survive their low glucose environment by resting and waiting for pro-proliferative conditions. ${ }^{[19]}$

In addition, AMPK is thought to inactivate SREBP1, a critical transcription factor which is involved in lipid and carbohydrate metabolism through phosphorylation [Figure 1]. ${ }^{[20]}$ Bungard et al. reported that AMPK activates transcription through direct association with chromatin and phosphorylation of histone H2B at serine 36, placing AMPK-dependent H2B Ser36 phosphorylation in a direct transcriptional and 
chromatin regulatory pathway leading to cellular adaptation to stress. ${ }^{[21]}$

Recently, it was reported that AMPK also plays a critical role in preventing oxidative stress induced upon glucose deprivation. The authors delineated a previously unidentified mechanism involving well-known AMPK downstream targets ACC1 and ACC2. Upon AMPK phosphorylation of ACC1 and ACC2, NADPH consumption is decreased and used as an antioxidant rather than a fatty acid synthesis precursor [Figure 1]. ${ }^{[22]}$ Thus, the diverse function of AMPK and its substrates allows for multiple cellular strategies to alleviate metabolic stress.

\section{Protein phosphatase 2A}

$\mathrm{PP} 2 \mathrm{~A}$ is a major serine/threonine protein phosphatase responsible for over half of all phosphatase activity in the cell. ${ }^{[23]}$ The active form of PP2A is a heterotrimeric complex consisting of a scaffold (A), catalytic (C) and regulatory (B) subunit. Previously, PP2A was viewed as a promiscuous enzyme with little specificity because its $A$ and $\mathrm{C}$ subunits are ubiquitously expressed with only two isoforms each. It is now well-established that substrate specificity and subcellular localization are determined by the regulatory B subunit contained in the heterotrimeric complex, which consist of 16 isoforms allowing for over 60 unique complexes. ${ }^{[23]}$

PP2A also exists as an inactive complex consisting of the $\mathrm{C}$ subunit and the PP2A binding partner $\alpha 4{ }^{\left[{ }^{[24]}\right.} \alpha 4$ binds to the $\mathrm{C}$ subunit, inhibits its activity and prevents its degradation until an adaptive PP2A-A/B/C complex is formed in order to dephosphorylate a specific substrate. ${ }^{[24]}$ PP2A and PP2A-like phosphatases have critical roles in nutrient sensing in lower level organisms such as yeast. ${ }^{[25]}$ However, only recently has PP2A been linked to nutrient sensing in mammalian cells.

Yan et al. demonstrated that PP2A is regulated by cellular amino acid levels. Upon amino acid withdrawal, they showed that PP2A is activated to dephosphorylate MAP4K3, an activating kinase upstream of mTOR, at Ser170 to inhibit mTORC1 signaling and prevent protein translation. ${ }^{[26]}$ Interestingly, when amino acids were added back to the system PP2A activity decreased and Ser170 phosphorylation increased, indicating a dynamic adaptive role of PP2A in nutrient sensing. Recently, it was reported that PP2A is responsive to a specific amino acid, glutamine. In response to in vitro glutamine deprivation or low in vivo glutamine levels, a specific PP2A B subunit, $\mathrm{B} 55 \alpha$, is induced at the transcriptional level to form an adaptive PP2A complex in order to promote cell survival by allowing p53 activation [Figure 1]...$^{[12]}$ Interestingly, the $\mathrm{B} 55 \alpha$ induction and complex formation is greatly enhanced in cells overexpressing $\alpha 4$, suggesting an important role for $\alpha 4$ in promoting PP2A complex assembly. Of significance, $\alpha 4$ is overexpressed in many human cancers and the PP2A/ B55 $\alpha$-p53 signaling axis may explain why many cancers are resistant to low in vivo glutamine levels as well as glutaminase inhibitors. Although exciting progress has been made, further studies are required to fully understand and appreciate the dynamics and importance of protein phosphatases' role in cell signaling and nutrient sensing.

\section{ADAPTATIONTHROUGHTRANSCRIPTION FACTORS}

\section{p53}

A major sensor and effector of cellular stress is the tumor suppressor $\mathrm{p} 53$. This diverse transcription factor is regulated at the protein level through various post-translational modifications such as ubiquitination and phosphorylation. Because p53 is commonly mutated in cancer, early studies focused mainly on its role in activation of apoptosis and cell cycle arrest in response to hypoxia and deoxyribonucleic acid (DNA) damage. Recently, several groups have reported critical roles for $\mathrm{p} 53$ in nutritional stress responses. New evidence suggests that specificity of p53 transcriptional activation largely depends upon promoter affinity and availability. ${ }^{[27]}$ Therefore, similar signaling cascades upstream of p53 may promote different cellular fates depending on context.

As mentioned previously, it was demonstrated that activation of p53 in response to low glucose levels promoted cell adaptation through a cell cycle arrest check point. ${ }^{[19]}$ Besides glucose, p53 is also important for survival when other nutrients such as amino acids are low. It was recently demonstrated that, like glucose deprivation, withdrawal of glutamine also activated p53 in a pro-survival manner and cells deficient of p53 were less viable under glutamine deprivation compared with those harboring functional p53. ${ }^{[12]}$ In correlation with p53 activation, another study found that cells underwent p53-dependent senescence when in conditions of low glutamine though inhibition of malic enzymes (ME). ${ }^{[28]}$ Furthermore, another amino acid, serine, also leads to p53-induced cell survival when its levels are depleted [Figure 1]. ${ }^{[29]}$ Therefore, unlike numerous DNA-damage induced p53 signaling cascades that result in apoptosis, it appears that $\mathrm{p} 53$ is important for cell survival under broad nutrient restrictions.

In contrast to induction of genes that promote cell cycle arrest, p53 also activates other metabolic regulators in response to 
nutritional stress. For example, under glucose starvation p53 promotes expression of Sco2, which decreases the rate of glycolysis by up-regulating mitochondrial oxidative phosphorylation (OXPHOS) [Figure 1]. ${ }^{[30]}$ Moreover, also under glucose starvation, p53 activates transcription of an essential factor for adipocyte development and fat metabolism, Lpin1, which also decreases glycolysis by promoting fatty acid oxidation. ${ }^{[31]}$ Thus, p53 may promote multiple mechanisms depending on cellular context in order to obtain similar outputs.

Interestingly, p53 also plays a transcription-independent role in cellular adaption during nutrient stress. Up-regulation of the pentose phosphate pathway (PPP) is a hallmark of proliferating cells as it is essential for the production of lipid and nucleotide biosyntheses that are required for cells to divide. A study by Jiang et al. showed that p53 physically interacts with and inhibits activation of glucose-6-phosphate dehydrogenase, the first rate limiting step enzyme of the PPP; thus, severely down-regulating the PPP and preventing further proliferative advantages. ${ }^{[32]}$ It should be mentioned that p53 also contributes to PPP up-regulation via transcriptional activation of TP53-induced glycolysis and apoptosis regulator (TIGAR). ${ }^{[33]}$ TIGAR acts as a fructose-2,6-bisphosphatase and promotes the PPP to produce NADPH and ribose-5-phosphate for antioxidant function and nucleotide biosynthesis, respectively. Interestingly, it was recently reported that TIGAR can contribute to intestinal tumor growth as loss of TIGAR decreased tumor burden and increased survival in a mouse intestinal adenoma model. ${ }^{[34]}$ These studies suggest a dynamic and context specific role of p53 in regulating PPP activity.

Taken together, p53 plays a dual role in cell proliferation and tumor development with respect to nutritional stress. On one hand, p53 helps highly proliferative and cancer cells survive temporary periods of diverse nutrient deprivations by inhibiting apoptosis and promoting cell cycle arrest. ${ }^{[12,19,28,29]}$ On the other hand, p53 hinders these cells by preventing Warburg-like properties that otherwise would lead to significantly enhanced proliferative capabilities. ${ }^{[30-32]}$ These observations may be important for developing novel cancer therapies aimed at targeting nutrient availability as p53 is one of the most commonly mutated genes in human malignancies.

\section{Peroxisome proliferator activated receptor gamma coactivator-I}

The PGC-1 family includes three isoforms, PGC- $1 \alpha$, PGC-1 $\beta$ and PGC-1-related coactivator (PRC), which play an important role in the control of energy homeostasis as co-activators of transcription factors. PGC- $1 \alpha$ has critical roles in mitochondrial biogenesis, cellular respiration rates and metabolic substrate use via co-activation of the transcription factors $\operatorname{PPAR} \gamma$, nuclear respiratory factor-1 (NRF-1), NRF-2 and FOXO1. ${ }^{[35]}$ As mentioned previously, p53 senses metabolic stress and leads to cell survival upon nutrient restriction. Recently, it was demonstrated that PGC- $1 \alpha$ interacts with p53 in response to metabolic stress. Specifically, p53 recruits PGC- $1 \alpha$ in response to glucose deprivation [Figure 1]. This interaction modulates p53 trans-activation, which causes preferential activation of cell cycle arrest and metabolic target genes. ${ }^{[35]}$ Interestingly, upon longer glucose starvation, the $\mathrm{PGC}-1 \alpha-\mathrm{p} 53$ interaction is abrogated via ubiquitin-mediated degradation of PGC- $1 \alpha$, causing the induction of apoptosis. These results give insight into the dual role of p53 and may explain why p53 activation can promote cell survival or cell death in similar contexts. Another study demonstrated that a different PGC-1 family member, PRC, is also responsive to metabolic stress. Gleyzer and Scarpulla found that glucose deprivation resulted in increased PRC protein levels and increased PRC-dependent gene expression. ${ }^{[36]}$ Thus, it will be important in the future to investigate not only changes in transcription factor levels in response to different metabolic stresses, but also changes to the diverse and dynamic cofactors required for transcription factor specificity.

\section{TAp63}

New evidence has demonstrated that $\mathrm{p} 63$, a protein structurally and functionally related to p53, also plays a role in tumorigenesis and metabolism. Although the exact role of $\mathrm{p} 63$ in tumor suppression is still unclear, it appears that the isoform maintaining its transactivation domain (TAp63) functions as a tumor suppressor. In contrast, the isoform lacking the transactivation domain may have oncogenic capabilities, including inhibition of p53 in a dominant-negative fashion. Indeed, mice deficient of TAp63 develop spontaneous, metastatic tumors. ${ }^{[37]}$ New studies by $\mathrm{Su}$ et al. have uncovered an interesting role of TAp63 in lipid and glucose metabolism. Their work showed that TAp63 acts as a master transcriptional activator of important metabolic regulators such as AMPKo2, Sirt1 and LKB1. ${ }^{[38]}$ Taken together, these data indicate that TAp63 is activated in response to metabolic stress and loss of this response may lead to tumor development.

\section{Perspective}

Metabolic reprogramming is a dynamic process requiring activation of specific proteins and genes at specific times. When these processes become unchecked, the potential 
for tumorigenesis increases dramatically. In the context of metabolism, this generally means an unnatural increase in nutrient uptake to support aberrant growth. However, the local supply of nutrients is not always constant; therefore, cancer cells frequently encounter acute or prolonged periods of nutrient deprivation.

Exciting progress has been made in understanding how cancer cells sense and adapt to ever changing nutrient conditions. The multifunctional roles of kinases and phosphatases in mammalian cells, such as AMPK and PP2A, exemplify how complex and intricate signaling pathways responsible for nutrient adaptation are. The downstream effectors, such as the transcription factor p53, are equally as complex in regulation of signaling networks. One reason the implication of p53 as a nutrient sensor is so exciting is that the classical paradigms of p53 status in tumors suggest that its loss is nothing but beneficial for tumors.

The recent demonstrations showing the critical role of p53 for cell survival under low glucose, low glutamine or low serine suggest that p53 may have a pro-tumor role and provides the foundation for targeting nutrient metabolism in p53 mutated/deficient tumors. The above work also suggests that to efficiently target nutrient-addicted cancers, it will be important to identify and understand the adaptive pathways used when nutrients are not available.

\section{REFERENCES}

I. Vander Heiden MG, Cantley LC, Thompson CB. Understanding the Warburg effect: The metabolic requirements of cell proliferation. Science 2009;324:1029-33.

2. DeBerardinis RJ, Lum JJ, Hatzivassiliou G, Thompson CB. The biology of cancer: Metabolic reprogramming fuels cell growth and proliferation. Cell Metab 2008;7:II-20.

3. Wise DR, DeBerardinis RJ, Mancuso A, Sayed N, Zhang XY, Pfeiffer HK, et al. Myc regulates a transcriptional program that stimulates mitochondrial glutaminolysis and leads to glutamine addiction. Proc Natl Acad Sci U S A 2008; 105:18782-7.

4. Wise DR, Thompson CB. Glutamine addiction:A new therapeutic target in cancer.Trends Biochem Sci 2010;35:427-33.

5. Nicklin P, Bergman P, Zhang B, Triantafellow E, Wang H, Nyfeler B, et al. Bidirectional transport of amino acids regulates mTOR and autophagy. Cell 2009;136:52I-34.

6. Shanware NP, Mullen AR, DeBerardinis RJ, Abraham RT. Glutamine: Pleiotropic roles in tumor growth and stress resistance. J Mol Med (Berl) 201।;89:229-36

7. Gao P, Tchernyshyov I, Chang TC, Lee YS, Kita K, Ochi T, et al. c-Myc suppression of miR-23a/b enhances mitochondrial glutaminase expression and glutamine metabolism. Nature 2009;458:762-5.

8. Yuneva M, Zamboni N, Oefner P, Sachidanandam R, Lazebnik Y. Deficiency in glutamine but not glucose induces MYC-dependent apoptosis in human cells. J Cell Biol 2007; 178:93-105.

9. Son J, Lyssiotis CA, Ying H, Wang X, Hua S, Ligorio M, et al. Glutamine supports pancreatic cancer growth through a KRAS-regulated metabolic pathway. Nature 2013;496:10I-5.

10. Urasaki Y, Heath L, Xu CW. Coupling of glucose deprivation with impaired histone H2B monoubiquitination in tumors. PLoS One 2012;7:e36775.
II. Roberts E, Frankel S. Free amino acids in normal and neoplastic tissues of mice as studied by paper chromatography. Cancer Res 1949;9:645-8, 3.

12. Reid MA, Wang WI, Rosales KR, Welliver MX, Pan M, Kong M. The B55 $\alpha$ subunit of PP2A drives a 553 -dependent metabolic adaptation to glutamine deprivation. Mol Cell 2013;50:200-II.

13. Gwinn DM, Shackelford DB, Egan DF, Mihaylova MM, Mery A, Vasquez DS, et al. AMPK phosphorylation of raptor mediates a metabolic checkpoint. Mol Cell 2008;30:2। 4-26.

14. Inoki K, Zhu T, Guan KL. TSC2 mediates cellular energy response to control cell growth and survival. Cell 2003; I 15:577-90.

15. Browne GJ, Finn SG, Proud CG. Stimulation of the AMP-activated protein kinase leads to activation of eukaryotic elongation factor 2 kinase and to its phosphorylation at a novel site, serine 398.J Biol Chem 2004;279:|2220-3I.

16. Leprivier G, Remke M, Rotblat B, Dubuc A, Mateo AR, Kool M, et al. The eEF2 kinase confers resistance to nutrient deprivation by blocking translation elongation. Cell 2013;153:1064-79.

17. He C, Klionsky DJ. Regulation mechanisms and signaling pathways of autophagy. Annu Rev Genet 2009;43:67-93.

18. Kim J, Kundu M, Viollet B, Guan KL. AMPK and mTOR regulate autophagy through direct phosphorylation of UlkI. Nat Cell Biol 20I I; I3:I32-4I.

19. Jones RG, Plas DR, Kubek S, Buzzai M, Mu J, Xu Y, et al. AMP-activated protein kinase induces a p53-dependent metabolic checkpoint. Mol Cell 2005; | 8:283-93.

20. Li Y, Xu S, Mihaylova MM, Zheng B, Hou X, Jiang B, et al. AMPK phosphorylates and inhibits SREBP activity to attenuate hepatic steatosis and atherosclerosis in diet-induced insulin-resistant mice. Cell Metab 201 1;13:376-88.

21. Bungard D, Fuerth BJ, Zeng PY, Faubert B, Maas NL,Viollet B, et al. Signaling kinase AMPK activates stress-promoted transcription via histone $\mathrm{H} 2 \mathrm{~B}$ phosphorylation. Science 2010;329:1201-5.

22. Jeon SM, Chandel NS, Hay N. AMPK regulates NADPH homeostasis to promote tumour cell survival during energy stress. Nature 2012;485:66I-5.

23. Janssens $\mathrm{V}$, Goris J. Protein phosphatase 2A: A highly regulated family of serine/threonine phosphatases implicated in cell growth and signalling. Biochem J 200I;353:417-39.

24. Kong M, Ditsworth D, Lindsten T, Thompson CB. Alpha4 is an essential regulator of PP2A phosphatase activity. Mol Cell 2009;36:5I-60.

25. Tate JJ, Feller A, Dubois E, Cooper TG. Saccharomyces cerevisiae Sit4 phosphatase is active irrespective of the nitrogen source provided, and Gln3 phosphorylation levels become nitrogen source-responsive in a sit4-deleted strain.J Biol Chem 2006;281:37980-92.

26. Yan L, Mieulet V, Burgess D, Findlay GM, Sully K, Procter J, et al. PP2A T6I epsilon is an inhibitor of MAP4K3 in nutrient signaling to mTOR. Mol Cell 2010;37:633-42.

27. Kracikova M, Akiri G, George A, Sachidanandam R, Aaronson SA. A threshold mechanism mediates $p 53$ cell fate decision between growth arrest and apoptosis. Cell Death Differ 2013;20:576-88.

28. Jiang $P$, Du W, Mancuso A, Wellen KE, Yang X. Reciprocal regulation of p53 and malic enzymes modulates metabolism and senescence. Nature 2013;493:689-93.

29. Maddocks OD, Berkers CR, Mason SM, Zheng L, Blyth K, Gottlieb E, et al. Serine starvation induces stress and $\mathrm{p} 53$-dependent metabolic remodelling in cancer cells. Nature 2013;493:542-6.

30. Mauro C, Leow SC, Anso E, Rocha S, Thotakura AK, Tornatore L, et al. NF-KB controls energy homeostasis and metabolic adaptation by upregulating mitochondrial respiration. Nat Cell Biol 201 I; 13:1272-9.

31. AssailyW, Rubinger DA,Wheaton K, Lin Y,MaW, XuanW, et al.ROS-mediated p53 induction of Lpin I regulates fatty acid oxidation in response to nutritional stress. Mol Cell 20I I;44:49I-50I.

32. Jiang $P$, Du W, Wang $X$, Mancuso A, Gao $X$, Wu $M$, et al. $p 53$ regulates biosynthesis through direct inactivation of glucose-6-phosphate dehydrogenase. Nat Cell Biol 20I I;13:310-6.

33. Bensaad K, Tsuruta A, Selak MA, Vidal MN, Nakano K, Bartrons R, et al. TIGAR, a p53-inducible regulator of glycolysis and apoptosis. Cell 2006; 126:107-20.

34. Cheung EC, Athineos D, Lee P, Ridgway RA, Lambie W, Nixon C, et al. TIGAR is required for efficient intestinal regeneration and tumorigenesis. Dev Cell 20I3;25:463-77. 
35. Sen N, Satija YK, Das S. PGC-I $\alpha$, a key modulator of $p 53$, promotes cell survival upon metabolic stress. Mol Cell 201 I;44:62I-34.

36. Gleyzer N, Scarpulla RC. PGC-I-related coactivator (PRC), a sensor of metabolic stress, orchestrates a redox-sensitive program of inflammatory gene expression.J Biol Chem 201 I;286:397| 5-25.

37. Su X, Chakravarti D, Cho MS, Liu L, Gi YJ, Lin YL, et al.TAp63 suppresses metastasis through coordinate regulation of dicer and miRNAs. Nature 2010;467:986-90.

38. Su X, Gi Y], Chakravarti D, Chan IL, Zhang A, Xia X, et al. TAp63 is a master transcriptional regulator of lipid and glucose metabolism. Cell Metab 2012;16:5। I-25.
How to cite this article: Reid MA, Kong M. Dealing with hunger: Metabolic stress responses in tumors. J Carcinog 2013;12:17.

Source and Support: Research is supported by a Stand Up To Cancer-American Association for Cancer Research Innovative Research Grant (SU2C-AACR-IRG0411), Stop Cancer Foundation, and the Sidney Kimmel Foundation for Cancer Research. MK is the Pew Scholar in the Biomedical Sciences. Conflict of Interest: None declared.

\section{AUTHOR'S PROFILE}

Michael A. Reid, Department of Cancer Biology, Beckman Research Institute of City of Hope Cancer Center, Duarte, CA 91010, USA

Mei Kong, Department of Cancer Biology, Beckman Research Institute of City of Hope Cancer Center, Duarte, CA 91010, USA
Journal of Carcinogenesis is published for Carcinogenesis Press by Medknow Publications and Media Pvt. Ltd.

Manuscripts submitted to the journal are peer reviewed and published immediately upon acceptance, cited in PubMed and archived on PubMed Central. Your research papers will be available free of charge to the entire biomedical community. Submit your next manuscript to Journal of Carcinogenesis.

www.journalonweb.com/jcar/ 